\title{
Three-dimensional sonographic measurement of normal fetal brain volume during the second half of pregnancy
}

\author{
Nanette M. Roelfsema, MD, ${ }^{a, *}$ Wim C. J. Hop, MSc, PhD, ${ }^{b}$

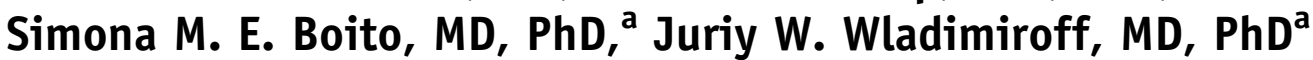

Departments of Obstetrics and Gynecology and Epidemiology and Biostatistics, ${ }^{\mathrm{b}}$ Erasmus MC, University Medical Center Rotterdam, Rotterdam, The Netherlands

Received March 18, 2003; revised June 26, 2003; accepted July 15, 2003

\section{KEY WORDS \\ Three-dimensional \\ Ultrasonography \\ Fetus \\ Brain volume \\ Brain weight}

\begin{abstract}
Objectives: This study was undertaken to develop a three-dimensional (3D) ultrasound method of measuring fetal brain volume.

Study design: Serial 3D sonographic measurements of fetal brain volume were made in 68 normal singleton pregnancies at 18 to 34 weeks of gestation. A comparison was made with fetal brain volume estimates from two-dimensional (2D) sonographic measurement of head circumference and published postmortem fetal brain weights.

Results: Coefficient of variation for fetal brain volume (3D) caused by differences between repeated tests was $10.2 \%$ and between analyses of the same recorded volume $2.2 \%$. Median brain volume increases from $34 \mathrm{~mL}$ at 18 weeks to $316 \mathrm{~mL}$ at 34 weeks. Median brain weight represented approximately $15 \%$ of total fetal weight. The $3 \mathrm{D}$ ultrasound-derived brain weight is larger than postmortem brain weight. However, this is not so for brain weight derived from total fetal weight at autopsy. A good agreement between 3D and 2D brain volume was found.

Conclusion: Sonographic measurement of fetal brain volume demonstrated an acceptable intraobserver variability and a nearly 10 -fold increase during the second half of gestation.

(c) 2004 Elsevier Inc. All rights reserved.
\end{abstract}

Both fetal biparietal diameter and fetal head circumference are standard parameters in establishing normal and abnormal fetal biometry. ${ }^{1}$ With the use of a threedimensional (3D) sonographic method, it would be possible to measure fetal brain volume. Recently, a method of $3 \mathrm{D}$ sonographic measurement of fetal liver volume

Extended funding was provided by the Dutch Organization for Scientific Research NWO.

* Reprint requests: N. M. Roelfsema, MD, Department of Obstetrics and Gynecology, Room 596, Erasmus MC-University Medical Center Rotterdam, PO Box 2040, 3000 CA, Rotterdam, The Netherlands.

E-mail: n.roelfsema@erasmusmc.nl was introduced, ${ }^{2-4}$ which would allow calculation of fetal liver to brain weight ratios in both normal and abnormal fetal development. ${ }^{5}$ Moreover, determination of the accuracy of a recently developed and simple two-dimensional (2D) sonographic method for estimating fetal brain volume, based on postmortem fetal brain weight data from literature, ${ }^{5}$ would be feasible.

The objectives of this study were therefore as follows: (1) to develop a $3 \mathrm{D}$ sonographic method of measuring fetal brain volume, (2) to establish reproducibility and normal data relative to gestational age, and (3) to compare 3D ultrasound data with $2 \mathrm{D}$ ultrasound data of brain volume and postmortem data of fetal brain weight at 18 to 34 weeks of gestation. 


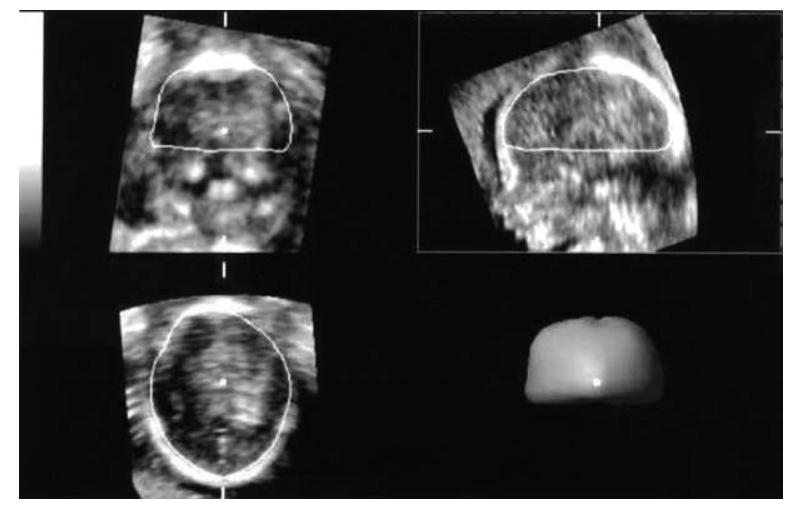

Figure 1 Measurement of fetal brain volume with 3D ultrasound.

\section{Material and methods}

\section{Study design}

During the period of February 2000 through September 2001, 68 women with a normal singleton pregnancy consented to participate in the study, which was approved by the Hospital Ethics Review Board. Pregnancy duration varied between 18 and 34 weeks (median 26 weeks). Maternal age ranged between 19 and 38 years (median 29 years). Women were recruited from the antenatal department and regional midwifery services. Pregnancy duration was determined from the last reliable menstrual period, or in case of uncertainty, adjusted by ultrasound in the first trimester of gestation. Pregnancies were uneventful resulting in the delivery of a normally developed infant. According to the Kloosterman tables, ${ }^{6}$ $91 \%$ of the birth weights were situated between the 5 th and the 95th percentiles, adjusted for maternal parity and fetal sex. In each pregnancy, fetal brain volume was established four times at 3- to 5-week intervals.

Intraobserver variability was determined during the same gestational period in 22 normal singleton pregnancies, 5 of which also participating in the serial study of fetal brain volume.

\section{Recording technique}

3D sonographic examination of the fetal brain was performed with the use of a standard Voluson $530 \mathrm{D}$ (Kretztechnik AG, Zipf, Austria) with a 3- to 5-MHz annular array transducer (VAW 3-5). An internal mechanism in the transducer sliced through the images and recorded a truncated pyramidal volume. Depth, longitudinal, and transversal dimensions were adjustable. An opening angle of 50 to 70 degrees and a sampling angle of 30 to 85 degrees was used, resulting in a maximum volume of 3.2 L. The depth range for the region of interest was set at 6 to $13 \mathrm{~cm}$. "Normal" frequency range (mid resolution/ mid penetration) was used in most patients, but was adjusted to "penetration" (lower resolution/high penetra- tion) in case of obesity. Frequency range "resolution" was used in case of thin women and/or superficial position of the fetus. Scanning time for one recorded volume ranged between 4 and 8 seconds, depending on fetal movement and size of the recorded volume.

The 3D ultrasound data for measurement of brain volume were recorded by using a sagittal scan of the head. Acquisition of this scan started at the midsagittal view of the head with the fetus facing the transducer. All data were first stored and processed by the equipment and displayed in three perpendicular planes on the monitor. The volume data were then collected on a transportable magnetic disk for later analysis (Iomega Jaz).

Measurements were made with the 3D view program (Kretztechnik AG, version 4.0) on a personal computer with an Iomega Jaz drive. The 3D brain volume (milliliters) was measured by rotating the recorded volume until the midsagittal plane was displayed on the upper right panel. With the VOCAL mode (method for measuring volume) the internal borders of the head were traced manually with stepwise rotation of 30 degrees, taking the skull base (defined as the line between glabella and opisthion) as the lower border (Figure 1).

For 2D sonographic estimation of brain volume, the head circumference (HC) was measured in the plane described first by Campbell and Thoms. ${ }^{1}$ The $2 \mathrm{D}$ brain volume was calculated according to the formula: 1/ $2 \times 1 / 6 \pi(\mathrm{HC} / \pi)^{3}$, described by Boito et al. ${ }^{5}$

Total fetal weight was estimated using the Hadlock formula, ${ }^{7}$ which includes measurement of fetal head and upper abdominal circumference as well as femur length. For comparison with estimated fetal weight and with postmortem data, fetal brain weight was estimated by multiplying fetal brain volume measured with $3 \mathrm{D}$ ultrasound with brain specific gravity (1.04). ${ }^{8}$

All 2D and 3D ultrasound examinations and measurements were performed by one observer (N. R.).

The reproducibility study consisted of recording two volume data sets from the same fetus with a time interval of approximately 20 minutes. The first recorded data set was analyzed twice with a minimal time interval of 1 week.

\section{Statistical analysis}

Statistical analysis was performed on a personal computer with the SPSS version 10.1 (SPSS Corp, Chicago, Ill). Analysis of the reproducibility study consisted of nested analysis of variance to separate the within subjects variation in components caused by differences between repeated tests within patients and differences between analyses of the same recorded volume.

Visual inspection of the scatter plot of 3D fetal brain volume versus gestational age showed roughly quadratic curves for individuals. Therefore, a linear relationship between the square root transformation of $3 \mathrm{D}$ brain 
volume versus gestational age was analyzed with regression analysis for repeated measurements (random coefficients model) with the use of SAS PROC MIXED (SAS Institute, Cary, NC). Weight-specific reference intervals were also calculated according to this model. The random coefficients model was also used for determination of the relationship between fetal brain weight (3D) versus estimated fetal weight and fetal brain weight as a percentage of total estimated fetal weight versus gestational age.

The agreement between both 3D and 2D fetal brain volume was assessed by calculation of the intraclass correlation coefficient (ICC). Systematic differences between 3D and 2D brain volume outcomes were calculated by means of the paired Student $t$ test. The $95 \%$ confidence limits of $3 \mathrm{D}$ fetal brain weight data were calculated for comparison with published postmortem brain weight data relative to gestational age ${ }^{9-11}$ and for comparison with published postmortem brain weight relative to total fetal weight. ${ }^{9,12}$

A $P$ value of less than .05 was considered significant.

\section{Results}

\section{D brain volume measurement}

A complete set of four 3D fetal brain volume measurements was collected in 65 of 68 women. A recording from the last scan was not available in 2 women and the last scan was not performed in the remaining woman, resulting in 269 recordings for 3D fetal brain volume measurement. A technically successful measurement was obtained in $245(91 \%)$ of 269 recordings. The remaining 24 recordings were of insufficient quality mostly due to the limited size of the recorded volume or position of the fetal head.

The reproducibility study showed a coefficient of variation for $3 \mathrm{D}$ fetal brain volume measurement of $10.2 \%$ caused by differences between repeated tests within women and $2.2 \%$ caused by differences between analyses of the same recorded volume.

Fetal brain volume data demonstrate a statistically significant linear increase for the square root transformation of fetal brain volume relative to gestational age (Figure 2). The median value (50th percentile) increases from $34 \mathrm{~mL}$ at 18 weeks to $316 \mathrm{~mL}$ at 34 weeks of gestation (Table I). Weekly increase (50th percentile) in fetal brain volume varies between $9 \mathrm{~mL}(28 \%)$ at 19 weeks to $26 \mathrm{~mL}(9 \%)$ at 34 weeks of gestation (Table I).

The relationship between the log transformation (base 10) of brain weight as a percentage of total fetal weight and gestational age appeared nonlinear as evidenced by a significant quadratic fitted curve $(P<.001)$ (Figure 3). Median fetal brain weight as a percentage of total fetal weight varies between $14 \%$ and $17 \%$ with

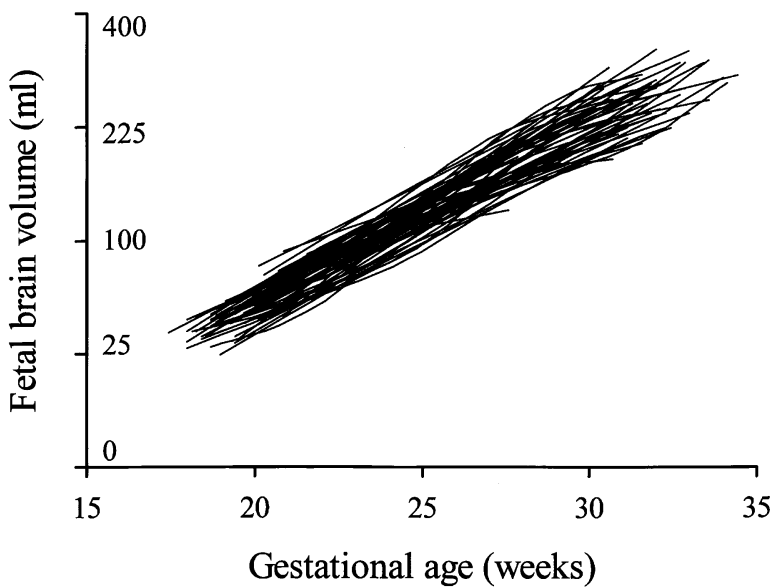

Figure 2 Longitudinal representation of fetal brain volume (in milliliters) relative to gestational age (in weeks). Data points (mostly four) are connected by straight line segments for individual fetuses. Note the square root-scaled vertical axis.

a decrease during the first half of the third trimester of pregnancy (Table I, Figure 3).

A clear difference exists between median 3D fetal brain weight and postmortem brain weights, which increases with gestational age (Table II).

When related to estimated fetal weight, mean 3D sonographic fetal brain weight agreed well with brain weight based on equations according to postmortem data from Guihard-Costa et $\mathrm{al}^{9}$ and Jordaan and Dunn ${ }^{12}$ (Table III).

\section{D brain volume measurement}

A 2D brain volume measurement was successful in 269 $(99 \%)$ of 271 recordings. Mean difference in fetal brain volume between the 3D and 2D sonographic measuring technique was $12 \mathrm{~mL}$, which is statistically significant $(P<.001)$. However, the overall agreement between 3D and 2D measurements of fetal brain volume was good, with an ICC of 0.95 .

\section{Comment}

To the best of our knowledge, this is a first longitudinal study on fetal brain volume measured by $3 \mathrm{D}$ ultrasound. Measurements on fetal head circumference and Doppler studies on intracerebral blood flow have demonstrated the presence of fetal brain sparing in fetal growth restriction associated with impaired uteroplacental perfusion. ${ }^{1,13}$

The current study shows an acceptable intraobserver variability for differences between repeated tests within women and between analyses of the same recorded volume.

A nearly 10 -fold increase in fetal brain volume takes place during the second half of gestation. A similar rise was established by Endres and Cohen ${ }^{14}$ in a cross-sectional study design that used a different $3 \mathrm{D}$ ultrasound 
Table I Fetal brain volume (in Milliliters) relative to gestational age (5th, 50th, and 95th percentile), weekly increment in fetal brain volume, mean fetal brain weight as a percentage of estimated fetal weight

\begin{tabular}{|c|c|c|c|c|c|}
\hline GA (wk) & $5 \%(\mathrm{~mL})$ & $50 \%(\mathrm{~mL})^{*}$ & $95 \%(\mathrm{~mL})$ & Weekly increment $(\mathrm{mL})$ & $\begin{array}{l}\text { Median fetal brain } \\
\text { weight/estimated fetal weight }(\%)^{\dagger}\end{array}$ \\
\hline 18 & 21 & 34 & 49 & - & 16 \\
\hline 19 & 29 & 43 & 59 & $9(28 \%)$ & 16 \\
\hline 20 & 38 & 53 & 71 & $10(24 \%)$ & 16 \\
\hline 21 & 47 & 65 & 85 & $12(22 \%)$ & 16 \\
\hline 22 & 58 & 77 & 100 & $13(20 \%)$ & 17 \\
\hline 23 & 69 & 91 & 116 & $14(18 \%)$ & 17 \\
\hline 24 & 82 & 16 & 133 & $15(16 \%)$ & 17 \\
\hline 25 & 95 & 122 & 152 & $16(15 \%)$ & 17 \\
\hline 26 & 109 & 139 & 173 & $18(15 \%)$ & 17 \\
\hline 27 & 124 & 157 & 194 & $19(13 \%)$ & 17 \\
\hline 28 & 140 & 177 & 218 & $19(12 \%)$ & 16 \\
\hline 29 & 156 & 197 & 243 & $21(12 \%)$ & 16 \\
\hline 30 & 174 & 219 & 269 & $22(11 \%)$ & 16 \\
\hline 31 & 192 & 241 & 297 & $23(10 \%)$ & 15 \\
\hline 32 & 211 & 265 & 326 & $24(10 \%)$ & 15 \\
\hline 33 & 231 & 290 & 356 & $25(9 \%)$ & 15 \\
\hline 34 & 252 & 316 & 389 & $26(9 \%)$ & 14 \\
\hline
\end{tabular}

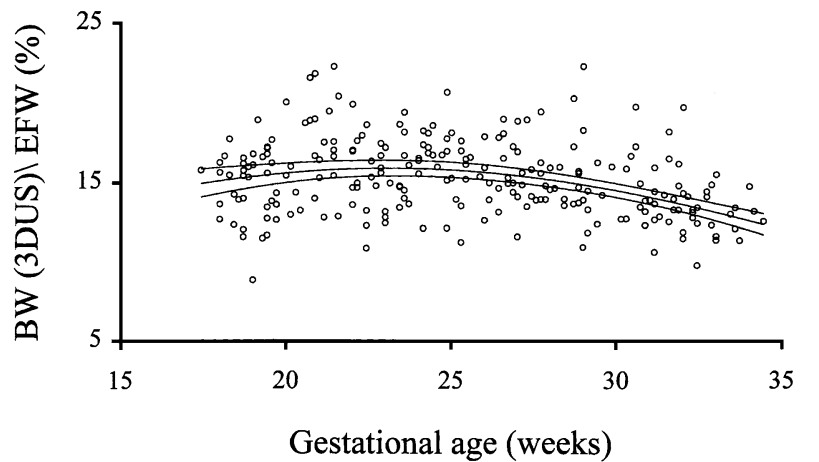

Figure 3 Fetal brain weight $(B W)$ as a percentage of estimated fetal weight $(E F W)$ relative to gestational age (in weeks). Curves represent fitted median values with $95 \%$ confidence limits.

method. At the same time, brain growth demonstrates a marked slow down as expressed by a weekly increment in brain volume at 34 weeks of only one third of the weekly increment at 19 weeks of gestation. When fetal brain weight derived from brain volume is examined, this represents $14 \%$ to $17 \%$ of total estimated fetal weight. Fetal brain weight expressed as a percentage of total fetal weight shows a significant reduction during the first half of the third trimester of pregnancy.

For producing a method that is easy to perform and is reproducible, it is necessary to take borders that are identifiable. The method we present here is based on the method described by Gordon in $1966 .{ }^{15}$ It concerns measurement of cranial capacity in children by means of radiography, taking the frontal pole (in this study "glabella") and occipital pole (or "opisthion") as the anterior and the posterior border, respectively. Gordon also includes those parts of the brain that are below the level of the skull base. However, in contrast with radiography, this part of the skull is not well visualized by ultrasound and could therefore not be measured. This could induce a small underestimation of the actual brain volume.

However, postmortem fetal brain weights were considerably lower than 3D sonographic fetal brain weights, particularly during the third trimester of pregnancy. These differences were less marked when fetal brain weight relative to total weight was taken into consideration, especially in the lower fetal weight classes. Several factors, such as cooling of the fetal specimen, cause of death, delivery-death interval, presence of traumatic, vascular, inflammatory, or other intracranial pathology, as well as the level at which the brainstem is sectioned, may influence the measured weight of the brain during autopsy. ${ }^{12}$ Moreover, fetal death may be associated with loss of water content that will affect fetal weight. This is supported by the observation that total body weight at autopsy is significantly lower than birth weight of live infants at a similar gestational age. ${ }^{11}$ This may also explain the good agreement between $3 \mathrm{D}$ sonographic and postmortem data when fetal brain weight relative to total weight is compared.

A good agreement, as expressed by an ICC of 0.95 , exists between $3 \mathrm{D}$ sonographic measurement and 2D sonographic estimation of fetal brain volume derived from postmortem fetal brain weight. The significantly smaller 
Table II Comparison of fetal brain weight (50th percentile) $(\mathrm{g})$ derived from the current 3D sonographic study and 3 postmortem studies

\begin{tabular}{|c|c|c|c|c|}
\hline \multirow[b]{2}{*}{ Gestational age (wk) } & \multicolumn{4}{|l|}{ Fetal brain weight $(\mathrm{g})$} \\
\hline & Current study (95\% confidence limits) & Guihard-Costa et al $^{9 *}$ & Singer et $\mathrm{al}^{10}$ & Gruenwald and Mingh ${ }^{11}$ \\
\hline 18 & $35(33-37)$ & 33 & - & - \\
\hline 20 & $55(53-57)$ & 48 & 49 & - \\
\hline 22 & $80(78-83)$ & 68 & 65 & - \\
\hline 24 & $110(107-113)$ & 91 & 83 & 92 \\
\hline 26 & $145(141-149)$ & 118 & 105 & 111 \\
\hline 28 & $184(179-189)$ & 147 & 132 & 139 \\
\hline 30 & $228(221-235)$ & 181 & 163 & 166 \\
\hline 32 & $277(268-286)$ & 219 & 198 & 209 \\
\hline 34 & $330(319-341)$ & 260 & 237 & 246 \\
\hline
\end{tabular}

* Data visually estimated from graphs in Guihard-Costa et al. ${ }^{9}$

Table III Comparison of fetal brain weight (50th percentile) (g) derived from the current 3D sonographic study and fetal brain weight from different equations or tables derived from published postmortem studies at the fetal weight range of $500-2500 \mathrm{~g}$

\begin{tabular}{llcl}
\hline & \multicolumn{2}{l}{ Fetal brain weight $(\mathrm{g})$} & \\
\cline { 2 - 4 } Fetal weight $(\mathrm{g})$ & $\begin{array}{l}\text { Current study* } \\
\text { (95\% confidence limits) }\end{array}$ & Guihard-Costa $^{9 \dagger}$ & Jordaan $^{*}$ and Dunn \\
\hline 500 & $80(78-83)$ & 80 & - \\
750 & $120(116-123)$ & 116 & 109 \\
1000 & $155(151-160)$ & 151 & 153 \\
1250 & $188(183-193)$ & 186 & 192 \\
1500 & $218(212-224)$ & 220 & 228 \\
1750 & $246(238-253)$ & 253 & 260 \\
2000 & $271(262-281)$ & 286 & 289 \\
2250 & $295(284-307)$ & 319 & 315 \\
2500 & $317(303-332)$ & 352 & 338 \\
\hline
\end{tabular}

$B W$, Fetal brain weight; $F W$, (estimated) fetal weight.

* Equation: $\log (\mathrm{BW})=-0.2453(\log \mathrm{FW})^{2}+2.3496 \log \mathrm{FW}-2.6499$.

${ }^{\dagger}$ Based on equation: $\log (\mathrm{BW})=0.92 \log \mathrm{FW}-0.58$.

$\ddagger$ Based on equation: $B W=543.85-(435.1452)(0.8077)^{\times} ; \times=(F W-750) / 500$; fetal weight $\geq 750 \mathrm{~g}$.

mean value (difference $12 \mathrm{~mL}$ ) for 2D sonographic brain volume estimates may be determined by the same limitations associated with postmortem fetal specimen as stated previously. ${ }^{12}$ Because measurement of fetal head circumference is part of routine biometry, the 2D sonographic method of estimating fetal brain volume described here is well applicable, although direct measurement of fetal brain volume with 3D ultrasound should be preferred.

Fetal brain volume measurement in conjunction with fetal liver volume determination could provide insight into the nature of abnormal fetal growth. Measurement of fetal brain volume beyond 34 weeks of gestation may be helpful in determining late onset growth retardation. However, there are several reasons for being increasingly less able of accurately establishing fetal brain volume by $3 \mathrm{D}$ ultrasound beyond 34 weeks of gestation. First, there are increasing limitations in recording the entire fetal head because of the limited 3D transducer sector size.
Second, near term, the fetal head is often situated deep down in the pelvic region, which makes it difficult to obtain the midsagittal plane necessary for correctly recording the volume. Third, the increased bone density of the skull leads to difficulties in visualizing the occipital border of the fetal brain.

It can be concluded that 3D sonographic measurement of fetal brain volume demonstrates an acceptable intraobserver variability. Fetal brain volume shows a nearly 10 -fold increase and represents $14 \%$ to $17 \%$ of total estimated fetal weight during the second half of gestation. Fetal brain weight as derived from 3D sonographic fetal brain volume measurements was higher than that obtained from postmortem fetal specimen. However, this difference becomes less evident when comparing fetal brain weight relative to total fetal weight. Whereas $2 \mathrm{D}$ and $3 \mathrm{D}$ sonographic volumes show a good agreement, 2D sonographic estimates were smaller at a mean difference of $12 \mathrm{~mL}$. 


\section{References}

1. Campbell S, Thoms A. Ultrasound measurement of the fetal head to abdomen circumference ratio in the assessment of growth retardation. BJOG 1977;84:165-74.

2. Chang FM, Hsu KF, Ko HC, Yao BL, Chang CH, Yu CH, et al. Three-dimensional ultrasound assessment of fetal liver volume in normal pregnancy: a comparison of reproducibility with twodimensional ultrasound and a search for a volume constant. Ultrasound Med Biol 1997;23:381-9.

3. Laudy JA, Janssen MM, Struyk PC, Stijnen T, Wallenburg HC, Wladimiroff JW. Fetal liver volume measurement by threedimensional ultrasonography: a preliminary study. Ultrasound Obstet Gynecol 1998;12:93-6.

4. Boito SM, Laudy JA, Struijk PC, Stijnen T, Wladimiroff JW. Three-dimensional US assessment of hepatic volume, head circumference, and abdominal circumference in healthy and growth-restricted fetuses. Radiology 2002;223:661-5.

5. Boito SM, Struijk PC, Ursem NTC, Wladimiroff JW. Fetal brain/ liver volume ratio and umbilical volume flow parameters relative to normal and abnormal human development. Ultrasound Obstet Gynecol 2003;21:256-61.

6. Kloosterman G. On intrauterine growth. Int J Obstet Gynaecol 1970;8:895-912.
7. Hadlock FP, Harrist RB, Sharman RS, Deter RL, Park SK. Estimation of fetal weight with the use of head, body, and femur measurements-a prospective study. Am J Obstet Gynecol 1985;151:333-7.

8. Duck FA. Mechanical properties of tissue. In: Duck FA, editor. Physical properties of tissue: a comprehensive reference book. London: Academic Press; 1990.

9. Guihard-Costa AM, Larroche JC, Droulle P, Narcy F. Fetal biometry: growth charts for practical use in fetopathology and antenatal ultrasonography. Introduction. Fetal Diagn Ther 1995;10:211-78.

10. Singer DB, Sung CJ, Wigglesworth JS. Fetal growth and maturation: with standards for body and organ development. In: Wigglesworth JS, Singer DB, editors. Textbook of fetal and perinatal pathology. Vol 1. Boston: Blackwell Scientific; 1991.

11. Gruenwald PM, Minh HN. Evaluation of body and organ weights in perinatal pathology. Am J Clin Pathol 1960;34:247-53.

12. Jordaan HV, Dunn LJ. A new method of evaluating fetal growth. Obstet Gynecol 1978;51:659-65.

13. Wladimiroff JW, Tonge HM, Stewart PA. Doppler ultrasound assessment of cerebral blood flow in the human fetus. BJOG 1986; 93:471-5.

14. Endres LK, Cohen L. Reliability and validity of three-dimensional fetal brain volumes. J Ultrasound Med 2001;20:1265-9.

15. Gordon IRS. Measurement of cranial capacity in children. Br J Radiol 1966;39:377-81. 